Dejan Todorović

Laboratory of Experimental Psychology

Department of Psychology

University of Belgrade
Original Scientific Paper

UDK 165 Витгенштајн Л.

159.937.51:165.3

\title{
WITTGENSTEIN'S ‘IMPOSSIBLE’ COLORS: TRANSPARENT WHITES AND LUMINOUS GRAYS
}

\begin{abstract}
In the book Remarks on Colors, Wittgenstein has claimed that transparent white objects do not and cannot exist, and that they cannot even be imagined. He had also claimed that luminous gray does not exist and cannot even be conceived. However, his arguments which aim to identify contradictory features of hypothetical transparent white media rely on incorrect assumptions about their properties and effects. Furthermore, some real objects and atmospheric phenomena can have features of transparent white media. As concrete examples of Wittgenstein's 'impossible' colors, this paper contains two simple computer-generated graphical displays, one depicting a scene that includes a transparent white sheet, and another which conveys the impression of luminous gray.
\end{abstract}

\section{Introduction}

Ludwig Wittgenstein has discussed color-related issues in a number of works throughout his life (Westphal, 2017). Some of his last notes on such topics were assembled by editors into a book called 'Remarks on color' (Wittgenstein et al., 1977). The book is divided into three parts (I, II, and III), but the order in which the material was written was probably II-III-I (Lugg, 2014b). In this paper I will discuss Wittgenstein's claims that two types of color impressions do not and cannot exist: transparent whites and luminous grays; note that 'converse' cases of transparent grays and luminous whites are well-known non-controversial phenomena. My discussion will be mainly based on remarks contained in Part I of the book, which probably presents the most mature expression of his thoughts on the subject (although many identical and very similar statements can be found in Part III as well, which I will occasionally also quote). I will not address the contents of some remarks in Part II which do not reappear in Part I, involving the 'cloudy' character of white. A thorough exposition of Wittgenstein's thoughts on transparent white was presented by Lugg (2014a).

Subsequent philosophical discussions of Wittgenstein's theses have generally neglected luminous grays and have mainly addressed the nontransparency of white. Accepting this claim as true, they differed as to whether 
its truth was part of the logic or grammar of colors (Horner, 2000; Gilbert, 1987; Gierlinger, 2009) or could be explained by some physical properties of whiteness and opacity (Westphal, 1986; Hardin, 1985). The only paper by psychologists dealing with Wittgenstein's remarks, to my knowledge, is by da Pos et al. (2014), who noted that psychological theories of perception of transparency do not necessarily rule out the existence of transparent whites, and claimed that transparent white objects, such as veils, do exist. In this paper I agree with da Pos et al. in this respect, but also present actual displays which can reasonably be described as depictions of transparent whites and luminous grays. Furthermore, I claim that Wittgenstein's analyses of effects of transparent media misrepresent some of their physical and perceptual aspects, rendering their conclusions invalid.

\section{Transparent Whites}

As a source of the claim that white cannot be transparent, Wittgenstein cites a letter of the painter Runge to Goethe, which contains the statement that there are transparent and opaque colors, and that white is opaque (see remark I-17 in Wittgenstein et al., 1977, p. 5a). Wittgenstein then asks 'Why is it that something can be transparent green but not transparent white?' (remark I-19). 'Green' here stands for any other chromatic color, such as red, yellow, blue etc., which can be transparent. White, together with black and shades of gray, belongs to achromatic colors; these are technical terms, not used by Wittgenstein. Note that his claim is not only that, as a matter of fact, there are no transparent white objects, but that there couldn't be such objects. He takes it for a fact that nothing can be transparent and white, and proceeds to look for an explanation of that fact. Furthermore, according to another remark, not only are transparent white objects non-existent and impossible, they are also unimaginable: 'Why can't we imagine transparent-white glass,- - even if there isn't any in actuality?' (remark I-31, p. 6e).

Wittgenstein's strategy to account for the non-existence of transparent white is to explicate rules governing the effects of transparent chromatic objects, to attempt to apply these rules to hypothetical transparent white objects, and to show that such a procedure cannot be consistently implemented. He writes: 'From the rule for the appearance of transparent coloured things that you have extracted from transparent green, red, etc., ascertain the appearance of transparent white! Why doesn't this work?' (remark I-29, p. 6e). 'Where does the analogy with transparent coloured glass go wrong?' (remark I-31, p. 6e).

Wittgenstein's explanation as to where the analogy breaks down is based on his understanding of how white and black surfaces are supposed to appear when observed through chromatic transparent media: 'Something white behind a coloured transparent medium appears in the colour of the medium, 
something black appears black.' (remark I-20, p. 5e). From the premise that a putative white transparent medium would have to produce effects analogous to effects of chromatic media, the following consequence is deduced: 'According to this rule, black on a white background would have to be seen through a 'white, transparent' medium as through a colorless one' (remark I-20, p. 5e). This reasoning is explicated in more detail in a remark from Part III: 'By analogy with the other colours, a black drawing on a white background seen through a transparent white glass would have to appear unchanged as a black drawing on a white background. For the black must remain black and the white, because it is also the colour of the transparent body, remains unchanged' (remark III-136, p. 34e-35e). Although Wittgenstein does not directly say so, this consequence of the assumption that transparent white media exist is clearly meant to be problematic: how could white and colorless transparent media have equal effects, in spite of the fact that white is certainly different from colorless, just as, for example, milk is different from water?

Another line of thought meant to lead to an unlikely conclusion is based on the following rule: 'Every coloured medium darkens that which is seen through it, it swallows light ... (remark I-30, p. 6e). From this premise an odd consequence follows: '... is my white glass also supposed to darken? And the more so the thicker it is? So it would really be a dark glass!' This is, obviously, another worrisome outcome, because white is certainly not dark. Some related, similarly head-scratching consequences of the assumption that transparent white exists are contained in Part III.

Given such counterintuitive conclusions, a reasonable move would be to deny the truth of some premises in the arguments. However, Wittgenstein never explicitly stated that, since the assumption that transparent white objects exist leads to absurdities, it is definitely proved that such objects cannot exist. As Lugg (2014a) noted, transparency of white seems to be a question 'Wittgenstein fails to wrestle to the ground' to his satisfaction. Nevertheless, in Part III he does ask is constructing a 'transparent white body' like constructing a 'regular biangle" (remark III-138, p35e), an object which is mathematically impossible.

Several philosophers who have discussed Wittgenstein's remarks on color have, in one way or other, supported the notion that transparent whites are not just empirically non-existent but logically inadmissible. For example, Westphal (1986) stated: 'I regard Wittgenstein's puzzle propositions (white is opaque ...) as necessary'. Gilbert (1987) claimed that 'We are seeking an explanation not only of why something can neither be both white and transparent nor look both white and transparent but also of why neither is even imaginable? Horner (2000) wrote about '... our intuitive feeling that the puzzle proposition [transparent white is impossible] is indeed an a priori necessary truth, that we as language users can know its truth in complete ignorance of any scientific theory'. Lugg (2014a) discussed the notion that the proposition 'no white 
surface is transparent' may be analytic, just as the proposition 'no bachelor is married'. In a similar vein, Gierlinger (2009) claimed that 'Asking why there is nothing transparent-white ... implies a grammatical claim about a certain understanding of "white" and "transparent". In opposition to such claims, in the following I will, first, argue that Wittgenstein's arguments against the existence of transparent white are based on false premises, second, claim that transparent white objects do exist, and third, provide a graphical depiction which evokes the impression of a transparent white object.

\section{Luminous Grays}

Wittgenstein devoted much less space to luminous gray than to transparent white, essentially just claiming that it does not exist, and that it cannot even be conceived. For example, he wrote: 'Whatever looks luminous, does not look gray' (remark I-36, p. 7e). 'For the fact that we cannot conceive of something 'glowing gray' belongs neither to the physics nor to the psychology of color' (remark I-40, p. 7e). So where does this fact belong? According to a remark in Part III, it belongs to the conceptual logic of color: 'That something which seems luminous cannot also appear grey must be an indication that something luminous and colorless is always called 'white'; this teaches us something about our concept of white' (remark III-217, p. 46e). Incidentally, Wittgenstein's use of the term 'colorless' in this quote is not felicitous, because white is not colorless in the way that a transparent glass is colorless; as noted before, the proper term in current use is 'achromatic'. It should be noted that, unlike for transparent white, he did not present actual arguments to show that the assumption of the existence of luminous gray would lead to absurd conclusions. In opposition to Wittgenstein's claim, here I will present a display which evokes the impression of luminous gray.

\section{The Basic Physics of Transparency and Wittgenstein's Analyses}

Objects in our environment can be opaque or transparent. When light falls on an opaque body, it is in part reflected and in part absorbed. Its color depends on the composition of reflected light, for example, its surface will generally look reddish if it reflects more light of longer wavelengths, and bluish if it reflects more light of shorter wavelengths. If light of all wavelengths is reflected more or less equally, the surface will look achromatic; in particular, it will generally look white if it reflects more than about $80 \%$ of incoming light. When light falls on a transparent body, it is also in part reflected and in part absorbed, but, in addition, it is in part transmitted through the body. An observer positioned in front of a transparent body will be able to see the scene behind the body, because light reflected from the scene is transmitted back through the body. 
As reported above, according to one of Wittgenstein's analyses a black and white scene when observed through a transparent white medium would have to look unchanged, because black surfaces would remain black and white surfaces would remain white; in other words, a white transparent medium would have the same (non)effect as a colorless transparent medium. Now, if this were true, it would be odd, but not necessarily impossible, because different structures and processes can in principle have equal effects. However, Wittgenstein's analysis has in fact a critical flaw: it is premised on one of his rules, which claims that black observed through a transparent colored medium would have to remain black. But, a glance through a transparent colored sheet of glass or cellophane teaches otherwise. Hold the sheet at a comfortable distance and look at a black surface, such that part of that surface is observed through the sheet and part of it is in free view. If the sheet is, say, green, then it will be readily seen that the part of the black surface observed through it looks different than the part in free view, in that the former part has a more or less salient greenish cast. The reason for this is simply that some light is reflected from the sheet; otherwise we would not be able to see the sheet itself, and would only see the portion of the scene behind it, inexplicably tinted green. Wittgenstein's notion that in such cases black would have to remain black does not seem to have been based on actual observations of scenes through colored transparent media, but rather on his conviction that a transparent glass can only 'swallow' light. This is wrong, since, as noted above, transparent media also reflect light to some extent. Therefore, the impression of the color of a surface behind a transparent medium is based not only on light reflected from that surface (as filtered by the medium), but also on light reflected from the medium itself (arriving from the same direction). In sum, the purported problematic equivalence of effects of transparent white media and transparent colorless media does not hold, and the corresponding deduction suggesting the impossibility of transparent white is invalid.

In the other analysis reported above, Wittgenstein claims that, first, a transparent white glass of increasing thickness would increasingly darken what is seen behind it, and second, that such a glass would therefore be dark. Several remarks are in order here. The first claim is not necessarily true, if 'darken' is taken to mean 'appears to have a darker shade'. For example, a white paper in shadow, or observed through sun glasses, although it sends a decreased amount of light towards the observer, does not appear to have a darker shade, such as, say, light gray, but rather still looks white. Such phenomena belong to color and lightness constancy, an important class of perceptual phenomena which I will not discuss here in any detail. It suffices to note that Wittgenstein is well aware of such effects, for example when he writes '... I see the parts of the paper that are farther away from the light as darker but still white, even though I would have to mix a grey to paint 
it' (remark III-171, p. 40e). The second claim is also not true. Note that transparent objects transmit, absorb, and reflect light, but differ with respect to percentages of transmittance, absorbance, and reflectance. It could be the case that increasing thickness of a transparent object might increase absorbance and decrease transmittance, but leave reflectance unchanged, and thus leave unchanged its perceived color, which depends on reflectance. On the other hand, it could also be the case that a transparent white object would indeed turn from white to gray with increasing thickness, similarly as a transparent red sheet might turn darker. In other words, a medium which is transparent white when thin might not be transparent white when thick. But if so, this would be irrelevant for the issue at hand: to resolve the question whether transparent white media could exist, it would be enough to show that thin transparent white media could exist. In sum, the possibility of a white glass turning darker while getting thicker doesn't render the existence of white glass per se impossible.

According to the above analyses, the assumption of the existence of transparent white media does not lead to absurdities. In other words, it has not been proven that transparent white is conceptually impossible. It could be the case, though, that transparent white is physically impossible. For example, Westphal (1986) has claimed that such a medium, being white, would have to reflect most incoming light, but, being transparent, would have to transmit most incoming light. However, it is not clear how much light a body would need to transmit in order to be regarded as transparent. Thus, a body could exist which reflects $80 \%$ of the light, enough for it to be white, and transmits most of the remaining $20 \%$, which might be enough for it to be transparent.

Now, even if it is the case that it has not been demonstrated that transparent white objects are conceptually or physically impossible, in the senses discussed above, it still might be the case that they could not exist for some other reason. It might also be the case that they could exist, but that for some reason they have never physically materialized. Or, they might even actually exist, but this may not be realized generally. This is in fact what I will claim below.

One way to counter the above criticisms would be to claim that they are thoroughly misguided, since they miss an overarching feature of Wittgenstein's approach to these issues, which involves the conceptual analysis of colors (also called their 'logic', 'grammar,' 'geometry', or 'geography'), rather than their physics or psychology. This type of critique was leveled at Westphal's (1986) physically based analyses (see Gilbert, 1987; Horner, 2000; Gierlinger, 2009). Regardless of whether such a general distinction of different approaches to color can be properly maintained, consider a concrete example, Wittgenstein's claim that black observed through a transparent medium remains black. How did he come to this conclusion? Is this statement arrived at solely on the basis of some insight into the 'grammar' of black and transparency? Whatever its 
origin, I have argued that it is wrong, and that Wittgenstein's arguments that rely on it are therefore wrong as well. Furthermore, this statement seems to be based on an incorrect assumption concerning some purely physical states of affairs, such as that transparent media only absorb light but don't reflect it. Such criticisms of crucial concrete aspects of Wittgenstein's analyses cannot be deflected simply by claiming that they miss the general tenor of his approach.

The basic phenomenology of transparency and Wittgenstein's analyses

The defining physical or optical characteristic of transparent media is that they transmit light. On the other hand, their basic visual or phenomenological characteristic is that observers can see scenes behind them; this is different from translucent media, which let light through but so thoroughly diffused to be spatially homogeneous, so that no scene is discernible behind them.

What is the relation between physical transparency and perception of transparency? Physically transparent bodies certainly often also look transparent. However, this is not necessarily the case. A transparent sheet lying flat on a uniformly colored background will not look transparent but opaque. Conversely, portions of non-transparent objects might look convincingly transparent, such as in photographs or paintings of transparent objects. Thus, to obtain an impression of transparency it is neither necessary nor sufficient to observe an actually physically transparent body. Rather, what is generally the case is that under standard observation conditions the physical characteristics of transparent media have some optical counterparts, which are interpreted by the visual system as indicators of transparency, and give rise to corresponding impressions.

Are there physical bodies that can generate impressions of transparent white media? Lugg (2014a) has listed quite a few potential examples, such as 'fog, frosted glass, muslin, ice on windshields, flour in a pail of water and tracing paper' (p. 206), but has claimed that Wittgenstein would have 'disparaged the widely-held view that there are no end of [such] examples of transparent white. However, actual refutations of legitimacy of such counterexamples for Wittgenstein's thesis cannot be found in Wittgenstein's text, and are missing in Lugg's exposition as well.

For a concrete example, consider materials with non-uniform structure, such as nets, consisting of a mesh of opaque fabric with holes. When observed from close up, the opaque portions of such materials can be clearly distinguished from the totally transparent holes, through which discrete portions a scene located behind can be observed. However, when observed from a far enough distance, or if the structure of the mesh is dense enough to be below visual resolution, one can obtain a convincing impression of a homogeneous transparent material through which a continuous scene can be seen. Such a material can have any color, including white. Thus, viewing a face through a white veil or a room behind a white curtain can be described as observing a scene behind a transparent white medium. Similarly, some 
instances of fog, mist, or smoke in a landscape can evoke impressions of inhomogeneous transparent white media.

A possible criticism of such proposed instances of transparent white media might claim that veils or smoke are not genuinely transparent, because they have complex, inhomogeneous and partly opaque structure. But what is tacitly assumed by such a critique is that a genuinely physically transparent medium would have to have uniform structure, such that any of its parts is equally transparent. However, on a microscopic level this is not true. For any material, on that level some of its portions consist of empty space, which simply lets light through, whereas other portions contain matter solid enough to absorb or deflect light. But even if this microscopic analogy were not fully appropriate, the fact remains that the above examples involve impressions of transparent white media. As such, they show that it is not true that transparent white is unimaginable or inconceivable. Wittgenstein stated that "When dealing with logic, "one cannot imagine that" means: one does not know what one should imagine here.' (remark I-27, p. 6e). But here one does know: just imagine a face behind a white veil or a landscape covered by a not too dense fog; or, even better, look at photographs of such scenes.

Which notion of transparency, physical or perceptual, would be more relevant for Wittgenstein's concerns? He did formulate some of his analyses in terms of physical properties of real and hypothetical transparent bodies. On the other hand, he mainly discussed only impressions of colors, rather than their corresponding physical properties. Interestingly, in Part III he asked 'What should the painter paint if he wants to create the effect of a white, transparent glass?' (remark III-198, p. 44e), and claimed that a strategy of painting a red transparent body and then substituting white for red would not work, because the impression of transparency would thereby be lost (see remark III-24, p. 19e). The fact that he addressed and discussed such issues shows that he regarded impressions of depictions of transparency as relevant for the problem of existence of transparent white.

\section{Graphical Depictions of Transparent White and Luminous Gray}

What are the optical conditions conducive for perception of transparency? Many studies of such issues have been carried out by perceptual psychologists, mainly using simple pictures, that is, physically non-transparent 2D configurations. Such research has identified conditions favorable and unfavorable for perception of transparency, such as geometric and photometric (dis)continuity of portions of the scene observed in free view and through the transparent sheet. For reviews see Kingdom (2011) and Gerbino (2013), and references listed there.

Figure 1, left, was constructed on the basis of insights from this literature. It takes up Wittgenstein's challenge of producing a painting (or rather a 
computer-generated display) of a transparent white object. Obviously, the display is not actually transparent, but it does look as a more or less convincing representation of a very simple scene which includes a transparent white rectangular sheet. The graphics itself is not particularly original, as displays similar or related to this figure are legion in the perceptual transparency literature, except that they mostly depict transparent gray or chromatic sheets; to my knowledge, no research has explicitly addressed the perceptually not particularly interesting issue of whether the depicted transparent body could look white.

As noted above, Wittgenstein claimed that one could not produce a painting of transparent white by starting from a painting of transparent red and then substituting white for red. Now, a reddish version of Figure 1, not presented here, can be easily produced by superimposing a red transparent filter, resulting in an image containing various tones of red (in fact only four different shades, for this simple image). If one would substitute the same white color for all the red shades, all sense of transparency would of course be lost, as the image would turn uniform white. The proper procedure, not mentioned by Wittgenstein, would not be to 'whiten' the original but to 'achromatize' it, by substituting appropriate shades of gray.

Figure 1, right, meets the challenge of depicting luminous gray. It is also not particularly original, having been constructed in the manner of figures published by Zavagno \& Caputo (2005), who explicitly intended to produce images portraying luminous gray. Like the depictions of transparency, impressions of luminosity are evoked by images that involve some optical characteristics of corresponding physical instances. In the case of perceived luminosity, the relevant visual feature is likely a visual halo, the gradient of light intensity surrounding a luminous body, as represented in the oval figures in the display. Incidentally, this display involves an intriguing visual illusion unrelated to the main issues in this paper, in that the surround in between the oval figures is physically uniform, but some of its portions look misty whereas others look plain.

Physically radiating bodies are often white, and their light intensity is much greater that the light intensity in the background. Such a strong light contrast is lacking in this figure, which may account for why the impression of luminosity is relatively weak. Such conditions are probably rather rare in everyday circumstances, and this empirical rarity rather than conceptual impossibility (as in Wittgenstein's claim, reported above, that 'something which seems luminous cannot also appear grey') could explain why the idea of luminous gray may seem odd. This oddness may teach us 'something about our concept of white, but only in the sense that our perceptual concepts are likely to reflect the statistical regularities in our environment, and how they are processed in the visual system. In other words, the 'grammar' of color is based on its physics, physiology, and psychology. 
Obviously, the displays in Figure 1 are neither actual white transparent sheets nor actual gray radiating light sources. However, they do constitute more or less cogent representations of such objects. As such, they do not provide proof that such objects really exist, because even impossible objects can have compelling graphical representations, as demonstrated masterfully by M.C. Escher. Nevertheless, they depict clearly and explicitly how such objects would look, providing compelling impressions of transparent white and luminous gray, and thus, together with the above criticisms of Wittgenstein's arguments, present challenges to the notion that such entities are non-existent, impossible and unimaginable.

Acknowledgement 1: This research was in part supported by grant OI179033 of the Ministry of Education, Science and Technological Development of the Republic of Serbia.

Acknowledgement 2: I wish to thank members and students of the Department of Philosophy at Belgrade University for constructive criticisms of an oral presentation of some of the material from this paper.

\section{References}

da Pos, O., Albertazzi, L., \& Villani, V. (2014). White Can Be Transparent: Why Wittgenstein is Wrong. Journal of the International Colour Association, 13, 84-90.

Gerbino, W. (2013). Achromatic Transparency. In J. Wagemans (Ed.) Oxford Handbook of Perceptual Organization. Oxford: Oxford University Press.

Gierlinger, F.A. (2009). The Philosophical Problem of Transparent-White Objects. In: V.A. Munz, K. Puhl, J. Wang (Eds.) Language and World. Papers of the 32nd International Wittgenstein Symposium. Kirchberg am Wechsel: ALWS.

Gilbert, P. (1987). Westphal and Wittgenstein on White. Mind, 96, 399-403.

Hardin, C.L. (1985). A Transparent Case for Subjectivism? Analysis, 45(2), 117-119.

Horner, E. (2000). 'There Cannot be a Transparent White': A Defense of Wittgenstein's Account of the Puzzle Propositions. Philosophical Investigations, 23(3), 218-241.

Kingdom, F.A. (2011). Lightness, Brightness and Transparency: A Quarter Century of New Ideas, Captivating Demonstrations and Unrelenting Controversy. Vision research, 51(7), 652-673. 
Lugg, A. (2014a). Wittgenstein on Transparent White. Wittgenstein-Studien, 5(1), 205-226.

Lugg, A. (2014b). When and Why Was Remarks on Colour Written-and Why is It Important to Know? In: Gierlinger, F.A., \& Riegelnik, Š. (Eds.) Wittgenstein on Colour. Berlin: de Gruyter

Westphal, J. (1986). White. Mind, 95, 311-328.

Westphal, J. (2017). Wittgenstein on color. In Glock, H.J., \& Hyman, J. (Eds.). A Companion to Wittgenstein. John Wiley \& Sons.

Wittgenstein, L., Anscombe, G.E.M., Macalister, L.L., \& Schättle, M. (1977). Remarks on Colour. Oxford: Blackwell.

Zavagno, D., \& Caputo, G. (2005). Glowing greys and surface-white: The photo-geometric factors of luminosity perception. Perception, 34(3), 261-274.
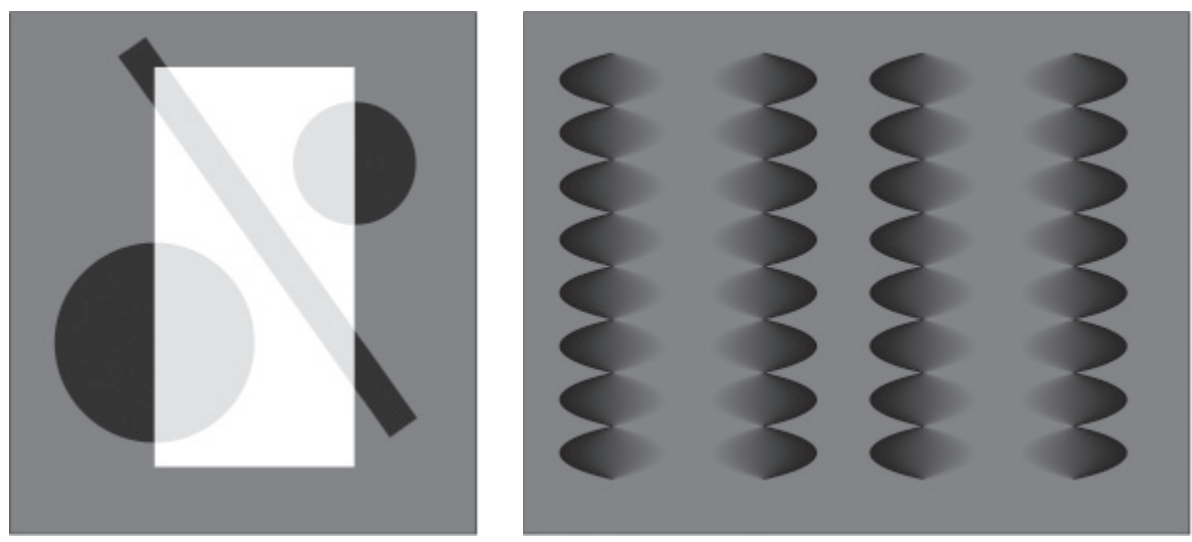

Figure 1. Left: A depiction of transparent white. Right: A depiction of luminous gray. 